\title{
An adaptive traffic MAC protocol based on correlation of nodes
}

\author{
Donghong $\mathrm{Xu}^{1,2}$ and Ke Wang ${ }^{1 *}$
}

\begin{abstract}
EA-MAC protocol is proposed on the basis of SMAC protocol to remedy the shortcomings of SMAC. In the EA-MAC, node correlation analysis algorithm and traffic adaptive duty cycle mechanism are added. Meanwhile, the residual energy is introduced into the existing traffic adaptive back-off mechanism to measure the saving efficiency. In the node correlation algorithm, all network nodes are divided into several areas by computing node correlation according to the collected information. The clustering mechanism is applied for choosing representative node in each area for transmitting data. This method can effectively reduce redundant nodes transmitting duplicate data. In traffic adaptive duty cycle mechanism, the duty cycle is regulated dynamically to decrease idle listening by comparing the threshold set with the flow value obtained from the predict flow model. In back-off mechanisms, by adjusting the value of contention window and back-off time, data collisions can be effectively avoided when network traffic is heavy. In addition, nodes with more remaining energy have priority to access the channel and have shorter back-off time, which can keep the balance of the whole network energy consumption and lengthen network lifetime. Simulation results show that the EA-MAC protocol has better energy saving, throughput, shorter delay performance, and low packet loss rate than that of SMAC protocol in dynamic traffic sensor networks.
\end{abstract}

Keywords: Wireless sensor networks, EA-MAC protocol, Node remaining energy, Traffic adaptive mechanism, Cross-layer optimization

\section{Introduction}

Current research in wireless sensor networks (WSN) mainly focus on data fusion, routing protocol, topology control, and medium access control (MAC) layer protocols $[1,2]$. The main objective is to reduce the energy consumption of the sensor network, increase throughput, and reduce the data transmission delay. $M A C$ protocol in data link layer determines the resource allocation of the radio channel, efficiently shares bandwidth resources by coordinating each node in network. Coordination performance directly determines the performance of the network, such as throughput, bandwidth utilization, fairness, or delay performance. According to resource allocation methods, $M A C$ protocol can be divided into three types: (1) contention-based $M A C$ protocol, (2) allocation-based $M A C$ protocol, and (3) mechanism-mixed $M A C$ protocol. $S M A C$ protocol $[3,4]$, which is a representative protocol

*Correspondence: Kewang330@163.com

1 School of Computer Science and Technology, China University of Mining and Technology, 221116 Xu Zhou, China

Full list of author information is available at the end of the article in contention-based $M A C$ protocol, is based on channel access competition mechanism. Based on IEEE802.11 $M A C$ protocol [5], SMAC protocol uses periodic listening/sleep working mode. This working mechanism has low duty-cycle and can reduce the power consumption by controlling non-working nodes to go into sleep state. But there are still some following drawbacks:

(1) Nodes are not differentially divided. In SMAC protocol, the nodes in the network use the same mechanism to work, but in reality, some information collected by nodes in the network is not reliable or redundant. In the context of reliable or redundant information, it may result in unnecessary waste of energy when letting all nodes involved in the channel contention. Therefore, it is better to selectively accept the information from partial nodes under certain conditions of network characteristics.

(2) Nodes cannot suit to the requirement of the dynamic changes of network traffic due to time for sleeping and listening being fixed for nodes through

\section{Springer}

(c) 2015 Xu and Wang Open Access This article is distributed under the terms of the Creative Commons Attribution 4.0 International License (http://creativecommons.org/licenses/by/4.0/), which permits unrestricted use, distribution, and reproduction in any medium, provided you give appropriate credit to the original author(s) and the source, provide a link to the Creative Commons license, and indicate if changes were made. 
periodic dormant and listening method in SMAC. Nodes cannot make the appropriate adjustments when large changes of network traffic occur. For example, when the network load suddenly increases, the listener time will become short and will result in multiple retransmissions of data; meanwhile, it will increase the chances of conflict and delay. When network traffic is low, energy maybe wasted due to the idle listening.

The paper [6-8] proposed a new schema to balance the lifetime of the deployed sensor network by assigning weights to links based on the remaining power level of individual nodes. Meanwhile, they developed a centralized heuristic to reduce its computational complexity. The methods and conclusion of the papers are taken as a basis for this paper. Han et al. [9] surveyed data communication protocols in DS-WSNS and the survey can be served as motivations and inspiration for our research. Xiao et al. [10] studied the underwater sensor networks according to the tight performance bounds of multihop fair access for $M A C$ protocols. The papers [11-13] proposed a novel compressive data collection scheme. The measurement methods give our basis for measuring our methods. In the research of channel access, He et al. [14] proposed a semi-random back-off method [15] that enables resource reservation in contention-based wireless LANs and the method can be readily applied to both 802.11.DCF and 802.11e EDCA networks with minimum modification.

For solving these two above discussed drawbacks, the paper proposes node correlation based on flow adaptive Efficient Adaptive Medium Access Control (EA-MAC) protocol to classify nodes by computing node correlation before data transmission. The algorithm for correlation computing between nodes can divide similar nodes to a certain area and choose the representative node for data transmission in the region. Representative node can cyclically be elected upon some parameters such as node residual energy and distance to sink node. During data transfer process, according to the prediction of flow changes, the system self-adaptively changes the duty cycle and contention window of the node. Conflict probability is significantly controlled by altering the back-off range and constraining the back-off window size, effectively reducing energy consumption.

The paper is organized as follows. In Section 2, we pose the problem and give the calculating processing. Meanwhile, $A R M A$ model for node flow forecasting is presented. In Section 3, we describe the EA-MAC protocol. In Section 4, the data and result of the experiment including average energy consumption, end-to-end delay, and effective throughput are given out. At last, we conclude with a summary and some directions for future work.

\section{Problem descriptions}

\subsection{Problem statement}

Wireless sensor networks (WSNs) emphasize data-centric approach for data transmission and the observer concerned merely indicator values measured within a monitored area rather than sensing data of some certain node. In general, node density of WSN is high. Therefore, in the same or similar monitoring area, these nodes have great similarity of data collection. Within a limited range of application, if only a certain number of nodes are selected for data transmission, the network life expectancy can be extended. Assuming there are 21 nodes deployed in a $100 \times 100 \mathrm{~m}$ area, with sink node as a data-receiving node, locating in the center, coordinates $(50,50)$ and the other 20 nodes are randomly distributed all around sink node. The concrete distribution can be seen in Fig. 1 .

\subsection{Calculation of correlation of nodes}

According to the above given example, within a certain range, node perception data have a certain degree of similarity. By calculating the entropy of each node, it indicates the average amount of information provided by a packet $[16,17]$. The joint entropy can be calculated by means of comparing the correlation entropy value with a given threshold $\epsilon$, to express node data correlation. Information entropy expressions are described as follows.

$$
\begin{aligned}
& H(x)=-\sum_{i=1}^{q} p\left(x_{i}\right) \times \log p\left(x_{i}\right) \\
& H(x)=-\sum_{i=1}^{q} p\left(y_{i}\right) \times \log p\left(y_{i}\right)
\end{aligned}
$$

In the above two formulas, $H(x), H(y)$ separately represent the entropy of node $i, j \cdot p\left(x_{i}\right)$ is the probability of events detected by the node i. $p\left(y_{j}\right)$ is the probability of events detected by the node $j$, and $q$ is the sum of all events which are being collected.

Joint entropy between node $i$ and node $j$ is expressed as follows:

$$
H(x, y)=-\sum_{i=1}^{n} \sum_{j=1}^{m} p\left(x_{i}, y_{j}\right) \log p\left(x_{j}, y_{j}\right)
$$

In formula $3, p\left(x_{i}, y_{j}\right)$ is the joint probability consisting of two-dimensional random variables $x_{i}, y_{j}$. In general, $H(x, y) \leq H(x)+H(y)$. If $H(x, y)=H(x)+H(y)$,which means information collected by two nodes are independent. $H(x, y) \backslash(H(x)+H(y))$ represents the correlation degree of data collection for $i$ th node and the $j$ th nodes, and $K$ is the correlation coefficient.

$$
K=1-\frac{H(x, y)}{H(x)+H(y)}
$$

Obviously, the data range of $K$ is [0:1]. $K=0$ indicates that data collected by node $i$ and node $j$ are independent. 


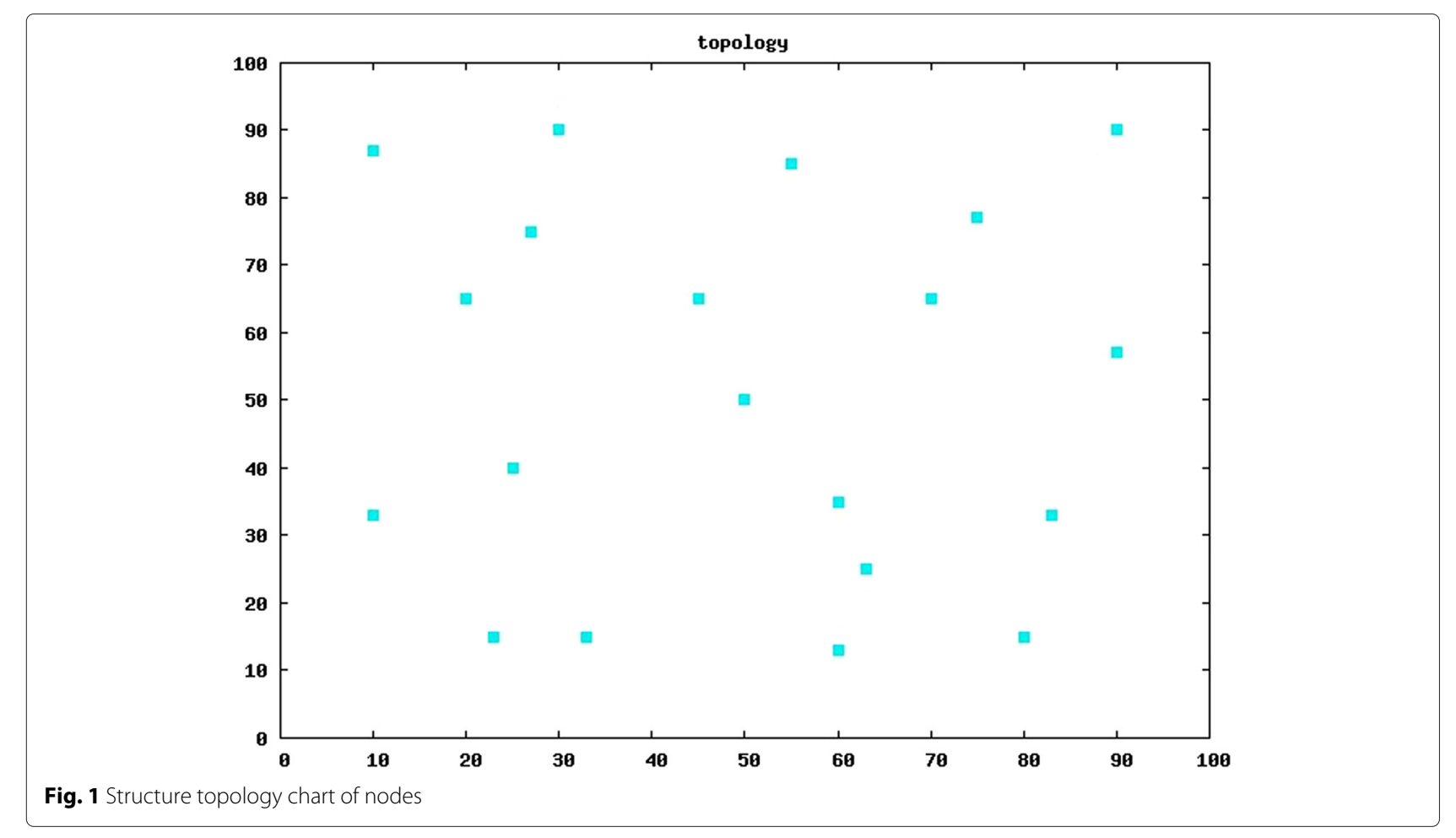

The larger the $K$ value, the greater the correlation between the two nodes. $K=1$ denotes that data collected by node $i$ and node $j$ are identical. Nodes are divided according to the correlation coefficient $K$ obtained, on the basis of meeting the requirements of the network application. The parameter $\epsilon=0.8$ is set as the threshold value. Information flow and its associating area can be sensed by node $i$. Node perceptual relevance schematic is shown in Fig. 2.

When $0<K \leq \epsilon$, the similarity of nodes is low. When $\epsilon<K \leq 1$, then the similarity of nodes becomes high. By calculating their joint entropy and their own information entropy, correlation coefficient between nodes can be obtained. The nodes satisfying $\epsilon<K \leq 1$ are divided into a group. All the nodes in the similarity coefficient are automatically divided into several smaller areas, called the relevant area (correlated area, CA). For each node within relevant area, cyclical formula 4 and 5 are used as merits of priority measure. The selected node within the relevant area is set as a representative node (RN) and cycle length is
$T$. The value of $T$ must be larger than and at least twice the value of the $E A-M A C$ protocol listener $\backslash$ sleep cycle to prevent the disruption of original data transmission caused by the switch of $R N$. Collected data will only be transmitted to sink node by $R N$.

\subsection{ARMA model for node flow forecasting}

Flow in the sensor network is usually dynamically changed with the practical applications and exhibits nonequilibrium with the position change of the nodes. Nodes nearby the base station bear heavy traffic. In some eventdriven applications, accident data stream is collected, while in the periodic data acquisition applications, network traffic is relatively stable. Therefore, the selection of the sensor network flow model must be consistent with its application scenarios. Literature [18-20] proposed an ARMA model for flow predictions, which applies to periodic data acquisition.

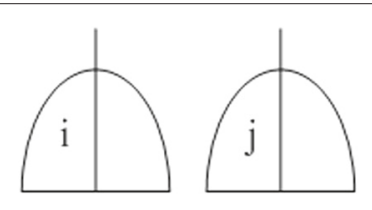

$K=0$

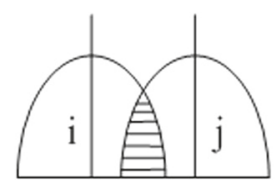

$0<K \leq \varepsilon$

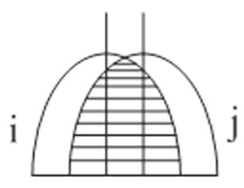

$\varepsilon<K<1$

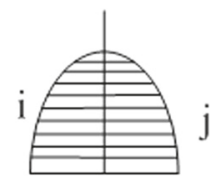

$K=1$

Fig. 2 Perceptual information correlation of nodes 


\subsubsection{ARMA model for periodic data applications}

Select the ARMA $(2 p, 2 p-1)$ model to analyze the flow of sensor network, where the positive integer $p$ is the order number. In order to avoid too much calculation, let $p=1$. Specific modeling process for $A R M A(2,1)$ is as follows: Assuming the sliding time window is $n$, the data traffic sequences $X_{0}^{\prime}, X_{1}^{\prime}, \cdots, X_{i}^{\prime}, X_{n}^{\prime}$ during each time slot do not appear smoothly and steady, so firstly taking the logarithmic to smooth it and getting the smooth sequences $X_{0}, X_{1}, \cdots, X_{i}, X_{n}$ and then creating $A R M A$ model with stationary series to predict the value of $n+1$ flow rate. Hence, we can know that a predicted value can be obtained for each forward sliding of the time window.

\subsubsection{Parameter selection}

When using $A R M A$ traffic forecasting model to predict the flow of nodes, firstly, a traffic acquisition cycle is necessary to be set. For example, if data are collected in $1 \mathrm{~s}$, the forecasting result is the next second flow. In most realtime applications, the acquisition cycle should be set to a smaller value to observe the flow changes timely. The ARMA model needs to calculate parameter values based on its historical data. During the periodic collection of data, the same data streams are generated as long as the transmission rate and the sleep/listening cycles of nodes are the same, so part of the data sequence is selected from [21-23]. In the paper, the parameters of the ARMA model are estimated as $\hat{\phi}_{1}=0.86579, \hat{\phi}_{2}=0.07356$, $\hat{\theta}_{1}=0.68954$, and $\hat{\sigma}_{a}^{2}=0.00186$. The advantage of $A R M A$ model is to provide accurate prediction of the actual flow of the node, and it is widely used for general periodic data collection. Traffic adaptive mechanism proposed in this paper applies flow $f_{\text {node }}$ prediction $A R M A$ model to adjust the duty cycle and the contention window.

\section{Description of EA-MAC protocol}

\subsection{Node classification strategy}

In the initial stage, for the election of first representative node in $T$ cycle, nodes are necessary to be synchronized. The sink node broadcasts the initialization message with maximum transmission power that covers the entire of radio network. After receiving the message, the sensor node begins to estimate its distance to the sink node based on the RSSI. Each sensor node has its separate scheduling table which contains all the scheduling information of all its nearby nodes. Node periodically broadcasts clock synchronization (SYNC) packet to coordinate the adjacent nodes to fit the scheduling period. After time synchronization, nodes begin to listen, compete for channel, and transmit monitoring data. When cycle $T$ terminates, nodes will enter the second stage of the relevant classification of nodes. Due to data having been collected in the preceding cycle $T$ (the number of events occurring in accordance with all the nodes within the cycle, and the number of sensed events for each node), the entropy and the correlation coefficient can be calculated for each node. After the correlation region is divided, an E-MSG message frame will be broadcasted using the frame format shown in Table 1.

Message field includes the frame type, node $I D$, frame size, residual energy, and the value of the distance (Dr) between the sink nodes. After receiving E-MSG message frame, the adjacent nodes within the relevant area, elect its representatives node $R N$ by following formula, where it denotes the balance factor.

$$
R I=E_{\mathrm{left}}^{\alpha} \times(D r)^{\beta}
$$

In the relevant area, the node with largest $R I$ value is selected as the representative node $R N$, which is responsible for the data transmission within the region. Other nodes will turn to sleep mode after selection and automatically start the timer, and participate in the next round of election. Flow chart for correlation algorithm is shown in Fig. 3.

The pseudocode of corresponding correlation algorithm is shown in Fig. 4.

\subsection{Self-adaptive algorithm of duty cycle}

During the periodic of sleep/listening, the value of duty cycle is changed with varying traffic conditions, known as adaptive duty cycle [24-26]. At present, many protocols made many improvements for adaptive duty cycle on the basis of $S M A C$ protocol. If the duty cycle is set in accordance with the low traffic load, greater delay will occur in large traffic condition. Node with higher data flow may result in blockage and collision due to the low duty cycle. By dynamically analyzing the network traffic, adjusting the duty cycle (i.e., when the network traffic load is large, the duty cycle is enlarged and when network traffic becomes smaller, the duty cycle is narrowed to accommodate the network traffic), the performance of entire network can be significantly improved in an abrupt flow pouring condition. In $E A-M A C$ protocol, two thresholds $L, H(L<H)$ are set for traffic prediction value $f_{\text {node }}$. The threshold value of duty cycle is $\left[D_{\min }, D_{\max }\right]$, which is set to $10 \%$ as a fixed value in the initial phase. (1) When the predicted value of the flow rate $\leq L$, the network traffic is low, the listener time and the duty ratio $D$ will further be reduced. Then, a new duty cycle will be obtained according to Eq. (6). If the new value of duty cycle is less than $D_{\min }, D_{\text {min }}$ will be replaced by $D_{\text {new }}$.

$$
D_{\text {new }}=D-\lambda \times H / f_{\text {node }}
$$

Table 1 E-MSG message frame structure

Frametype Node ID Framesize Node residual Distance between energy sink nodes 
Xu and Wang EURASIP Journal on Wireless Communications and Networking (2015) 2015:258

Page 5 of 13

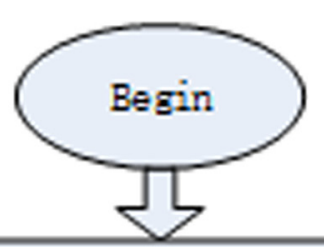

Sink Sending Initiation Message

Receiving Message, computing the Distance $(\mathrm{Dr})$, Starting the Timer $(\mathrm{T})$

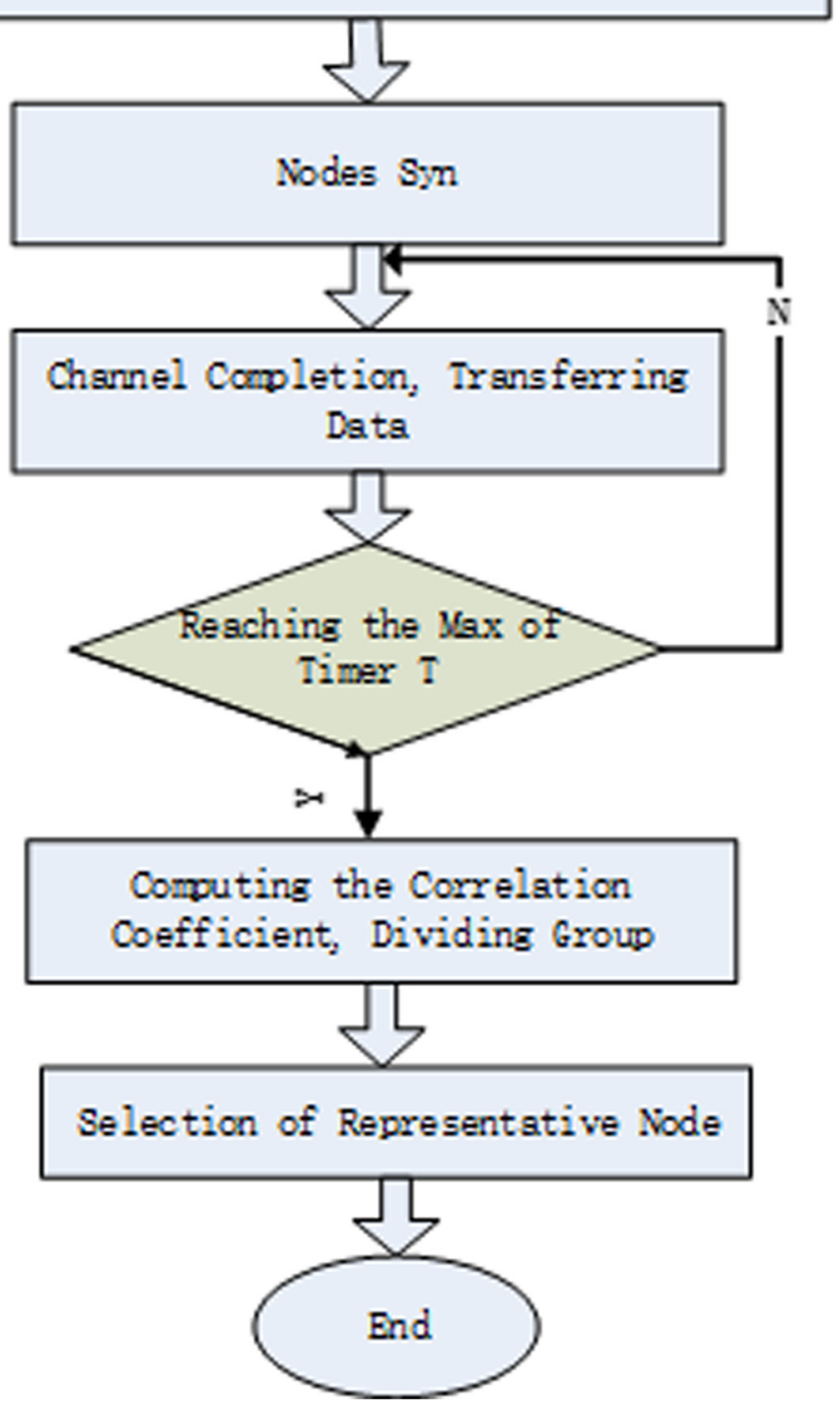

Fig. 3 Flow chart for correlation algorithm 


\section{Input: $\operatorname{Sink}$ Node, Nodes}

Output: Correlation Region, Representative Node*

\section{Begin}

2.Sink.Send(Initiation-message)

3.Nodes. Receive(message, SYNC)

4.Dr=Computing the Distance

\section{Starting-Timer(T)}

6.Syn-Nodes

\section{Lisenting(Channel)}

\section{Completing.Channel}

9. Transferring Monitoring Data

10.If $(T>\operatorname{Max})$

\section{Computing entropy according formula $1,2,3$}

\section{Computing Correlation-}

\section{Dividing-Different-Area}

14. Correlation Region- 4

\section{5. else goto 7}

16:Selection Representative Node

\section{End}

Fig. 4 Pseudocode of corresponding correlation algorithm

(2) When the flow predictive value $L<f_{\text {node }}<H$, network traffic is moderate, the duty cycle will be restored to its original size.

(3) When the flow predictive value $f_{\text {node }} \geq H$, the network traffic is large, the duty cycle will be increased into $D_{\text {new. }}$

$$
D_{\text {new }}=D+\lambda \times H / f_{\text {node }}
$$

Flow chart for duty cycle self-adaptive algorithm is shown as Fig. 5.

The pseudocode of duty cycle self-adaptive algorithm is shown as follows in Fig. 6 .

\subsection{Self-adaptive back-off algorithm}

In adapting mechanism of $C S M A / C A$ and competitionbased $M A C$ protocol, the exponential back-off algorithm 


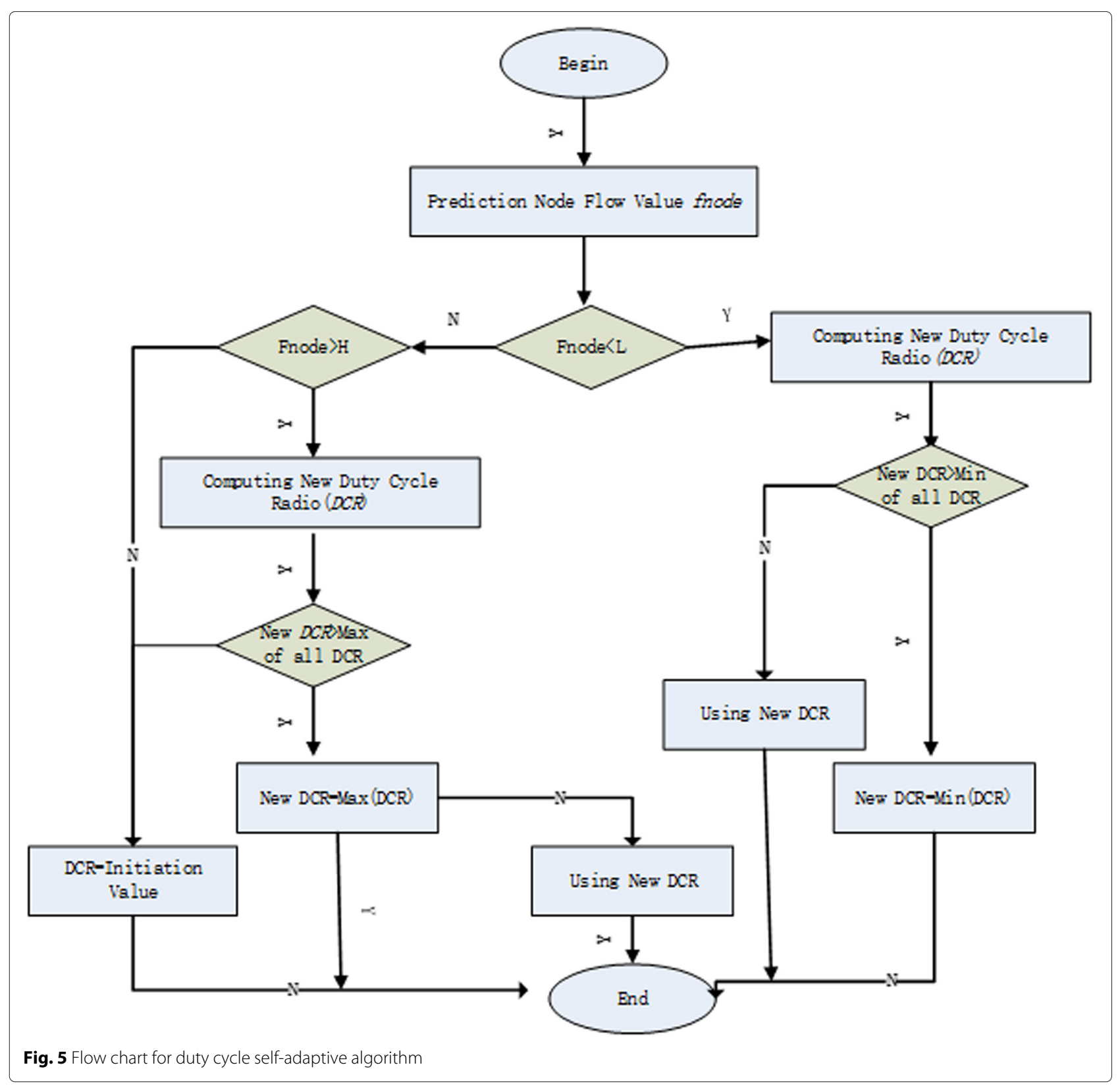

is an important component [27-29] and it decides the efficiency of $C S M A / C A$. Nodes need to follow a certain back-off algorithm to wait for resending data when the channel is listened to be occupied or clashed with other nodes. Back-off algorithms can be mainly divided into two types: binary exponential back-off algorithm (BEB) and multiple increase and linear decrease algorithm (MILD) $[28,29]$. Achieving the purpose of back-off is to reduce the conflict possibility when data are transmitted. The longer the back-off time is, the less chances that the channel being seized with other nodes becomes, which leads to the less likelihood of conflicts. Whereas the shorter the back-off time is, the more chances for short time channel seizing becomes, which leads to the larger potential for conflicts. Back-off value is calculated by the following formula:

$$
\text { BackoffTime }=\text { Random }() \times \alpha \text { SlotTime }
$$

In the above formula, $\alpha$ SlotTime is the fixed slot value in network. Random() is a uniformly distributed pseudorandom integer in the contention window [0, CW]. The lower limit value of $C W$ is $C W_{\min }$ and the upper limit is $C W_{\max }$.

(1) Selection of $C W_{\min }$ and $C W_{\max }$ 
Input: $L, H, D_{\min }, D_{\max }, f_{\text {node }}$

Output:-New·DCR

1.Begin

2.If $\left(f_{\text {node }}<L\right)$

3. $\{\cdot+$

4. Computing. DCR

5. If. $(C D R>M i n)$

6. $D C R=\operatorname{Min}(D C R)$

7.else.Using.New.DCR

8.

9. else $\cdot i f\left(f_{\text {node }}>H\right)$

10. $\{4$

11. New $\cdot D C R=$ Computing $\cdot D C R$

12. If. (New.DCR>Max)

13. New $\cdot D C R=\operatorname{Max}(D C R)$

14. else

15. using New'DCR

16.4

17. else

18. $\cdot D C R=$ Initiation $\cdot$ Value

19. End

Fig. 6 Pseudocode for duty cycle self-adaptive algorithm

For the selection of $C W_{\min }$ and $C W_{\max }$, node traffic information $f_{\text {node }}$ needs to be combined to adjust the contention window. When $f_{\text {node }}<L$, half the value of the $C W_{\min }$ and $C W_{\max }$ is given, thus reducing the waiting, the back-off time, and the delay time. When $f_{\text {node }}>H$, it increases the value of $C W_{\min }$ and 
$C W_{\max }$, thus reducing the back-off time for channel listening and collisions.

(2) Selection value of node $C W$

In $E A-M A C$ protocol, the back-off time for node accessing channel $(\mathrm{CW})$ obeys normal distribution. Mean value of normal distribution is related to $C W_{\min }$ and energy factor $\eta$. The variance of normal distribution is related to $C W_{\min }$. Let the initial energy value be $E_{\max }$; the node's current residual energy value is $E_{\text {left }}$ so the node's energy factor is $\eta=E_{\text {left }} / E_{\max }$. The value of mean and variance value for normal distribution are shown as follows:

$E(C W)=C W_{\min }(1-\eta)$

$$
D(C W)=C W_{\min } / 4
$$

In general, the slot is the smallest unit for $C W$ and in this paper, the entire time slot is selected by using rounded down approach. In addition, $C W$ also needs to meet certain ranges:

$$
C W_{\min } \leq C W \leq C W_{\max }
$$

where

$$
\begin{aligned}
& C W_{\min }=\left\lfloor\frac{10\left(C W_{\max }+1\right) \times(1-\eta)}{C W_{\min }+1}\right\rfloor \\
& C W_{\max }=\left\lfloor\frac{10\left(C W_{\max }+1\right) \times(\eta)}{C W_{\min }+1}\right\rfloor
\end{aligned}
$$

According to Eq. (9), it is clear that when the value of residual energy of the node is larger, the mean value of the normal distribution will be relatively smaller. Meanwhile, the probability of back-off time $C W$ to select a smaller value is larger. When the remaining energy is smaller, the mean value of the normal distribution will be relatively larger. Meanwhile, the probability that $C W$ achieves greater value is larger. From Eq. (10), it is obvious that $D(C W)$ selection is related to $C W_{\min }$. When the flow rate is increased, $C W_{\min }$ increases together, and the variance of the normal distribution becomes large, showing a large normal unevenness. So back-off time difference selected by the sending node is relatively large with minimal conflict potential. In this back-off method, back-off time selection is related to the normal distribution function in formulas 9 and 10, allowing the node with more residual energy access channel with priority, taking more responsibility for data transmission, thus balancing network energy distribution, and extending the network life cycle. When network traffic is large, the selection range of the back-off time is increased as well as the difference, reducing the possibility of simultaneous data sending. It is not necessary for the node to inform the neighbor nodes the remaining energy, thus avoiding the overhead of broadcast communication.

\section{Simulations}

NS2 platform [30, 31] is applied for network simulation to compare $E A-M A C$ and $S M A C$ protocol. Duty cycle of $S M A C$ protocol is set to $10 \%$. Listening sleep cycle is set to $1.6 \mathrm{~s}$, and listening time is set to $0.16 \mathrm{~s}$. Cycle $T$ for the relevant regional is selected to $6.4 \mathrm{~s}$, four times of the listener sleep cycle. Other parameter settings are shown in Table 2. In Table 2, ILPC stands for idle listening power consumption.

In the single-hop scene, packet interval is sent to change the variation of network traffic. With network traffic varying, the duty cycle for listening/sleep also alters. Delay and back-off time for channel listening vary with the residual energy and network traffic. The initial operation period of the network is $0-100 \mathrm{~s}$. The medium phase of network operation is $100-200 \mathrm{~s}$. For the period of $200-300 \mathrm{~s}$, transmission interval is reduced, and the network traffic is moderate. During $300-400 \mathrm{~s}$, the transmission interval is further reduced, and the network load is high. Late network operation period: 400-650 s, the transmission interval is increased, and the network is in a low flow state. Gawk [32] is used to run the simulation script files and analyze the generated trance file. It also can obtain delay, average energy consumption of the network and packet loss rate, and corresponding file. Simulation data is extracted with 10-s interval and plotted below.

\subsection{Average energy consumption}

From the test chart of Fig. 7, data sending time starts at the 100 s. $E A-M A C$ and $S M A C$ have consumed part of the energy, and the energy consumed in $E A-M A C$ is about $1 \mathrm{~J}$ higher than $S M A C$. This is because the $E A-M A C$ increases the relevance computing of nodes and network traffic assessments. During 100-200 s, the network is in a low flow rate, so $E A-M A C$ with adaptive duty cycle mechanism will promptly adjust its duty cycle rate, and thus the sleep time becomes long, resulting in smaller energy consumption than that of SMAC. During 200-300 s, with the decrease of transmission interval, network traffic begins to increase. At about $250 \mathrm{~s}, E A-M A C$ increases its own duty cycle and energy consumption. During 300-400 s, network traffic is high, and in $E A-M A C$, it continues to increase with duty cycle and energy consumption. During 400-650 s, network traffic in $E A-M A C$ is detected

Table 2 Parameter setting

\begin{tabular}{lccc}
\hline Packet size $(\mathrm{B})$ & 50 & $\mathrm{SIFS}(\mathrm{ms})$ & 5 \\
Sending power $(\mathrm{mW})$ & 360 & $\mathrm{DIFS}(\mathrm{ms})$ & 10 \\
Sending power $(\mathrm{mW})$ & 360 & $\mathrm{DIFS}(\mathrm{ms})$ & 10 \\
Receiving power $(\mathrm{mW})$ & 360 & $\mathrm{ILPC}(\mathrm{mW})$ & 340 \\
Sleep power consumption $(\mathrm{uW})$ & 50 & Routing protocol & AODV \\
Node initial energy $(J)$ & 100 & Simulation time $(\mathrm{s})$ & 650 \\
\hline
\end{tabular}




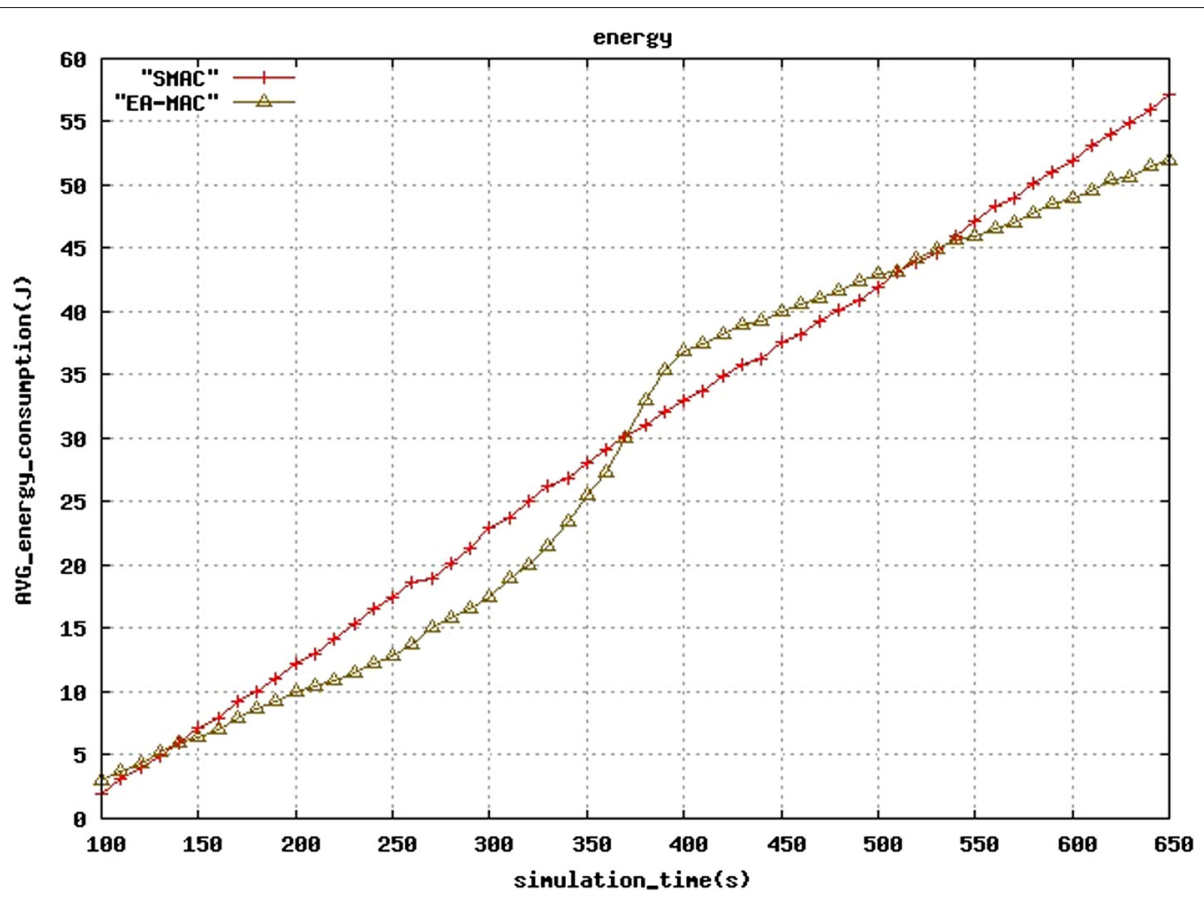

Fig. 7 Energy-efficiency consumption

in low state, thus duty cycle is reduced together with its energy consumption. From the beginning to the end, $S M A C$ protocol keeps constant duty cycle, with stable growth of energy consumption in earlier stage (flow is high). In the latter stage (flow is low), packets that accumulated in buffer queue start to be transmitted, so the energy consumption continues to increase. As can be seen from Fig. 7, when network traffic dramatic changes with high traffic, the advantages of $E A-M A C$ energy is more pronounced than that in the $S M A C$.

\subsection{End-to-end delay}

There are many factors to impact the delay of packet sending of $E A-M A C$, such as carrier sensing delay, back-off delay, transmission delay, sleep delay, and queuing delay. From Fig. 8, we can see that in 100-200 s, delay of $E A$ $M A C$ is higher than that of $S M A C$, due to the lower duty cycle of $E A-M A C$, more affection of sleep latency in low flow state. In $200-420 \mathrm{~s}$, the delay of the $E A-M A C$ is much lower than that of $S M A C$, because of the constant increase of duty cycle of $E A-M A C$ with increasing flow rate. In this case, large amounts of data do not accumulate in node queue, and packet queuing delay brought by the high flow is less affected. In comparison, SMAC in 100$400 \mathrm{~s}$ shows an opposite trend. Delay increases as network traffic rises. In 400-500 s, delays continue to increase; reaching its highest point at $500 \mathrm{~s}$ and a lot of data packets are discarded at that time due to exceeding the cache time limit. Then in low flow state, delay quickly restored to its previous state (lower level).

\subsection{Effective throughput}

As can be seen from Fig. 9, with the data transmission interval increases, network traffic is reduced. Both protocol throughputs are increasing at the last interval of $2.5 \mathrm{~s}$. Throughput value looks close because of the lowflow state and little network conflicts. Whereas when the network traffic increases, the throughput increases with the increase of network traffic, but because collision rate increases, the increasing rate of throughput value decreases. But it can be clearly seen from Fig. 9 that throughput of $E A-M A C$ protocol shows better performance in high-flow state than that of $S M A C$. The back-off mechanisms proposed in this protocol has an impact on energy consumption, latency, throughput, and packet loss rate based on flow state and varying residual energy. Differences of back-off range may reduce the likelihood of conflict when flow increases. Besides, the network load can be more balanced by arranging smaller back-off value to nodes with less residual energy to increase throughput and promote packet sending.

\subsection{Packet loss rate}

As can be seen in Fig. 10, weather $E A-M A C$ is the in low traffic flow or high traffic flow. It can keep the packet loss rate below $10 \%$. The reason is that with the increasing of traffic flow, $E A-M A C$ timely evaluates the traffic flow 


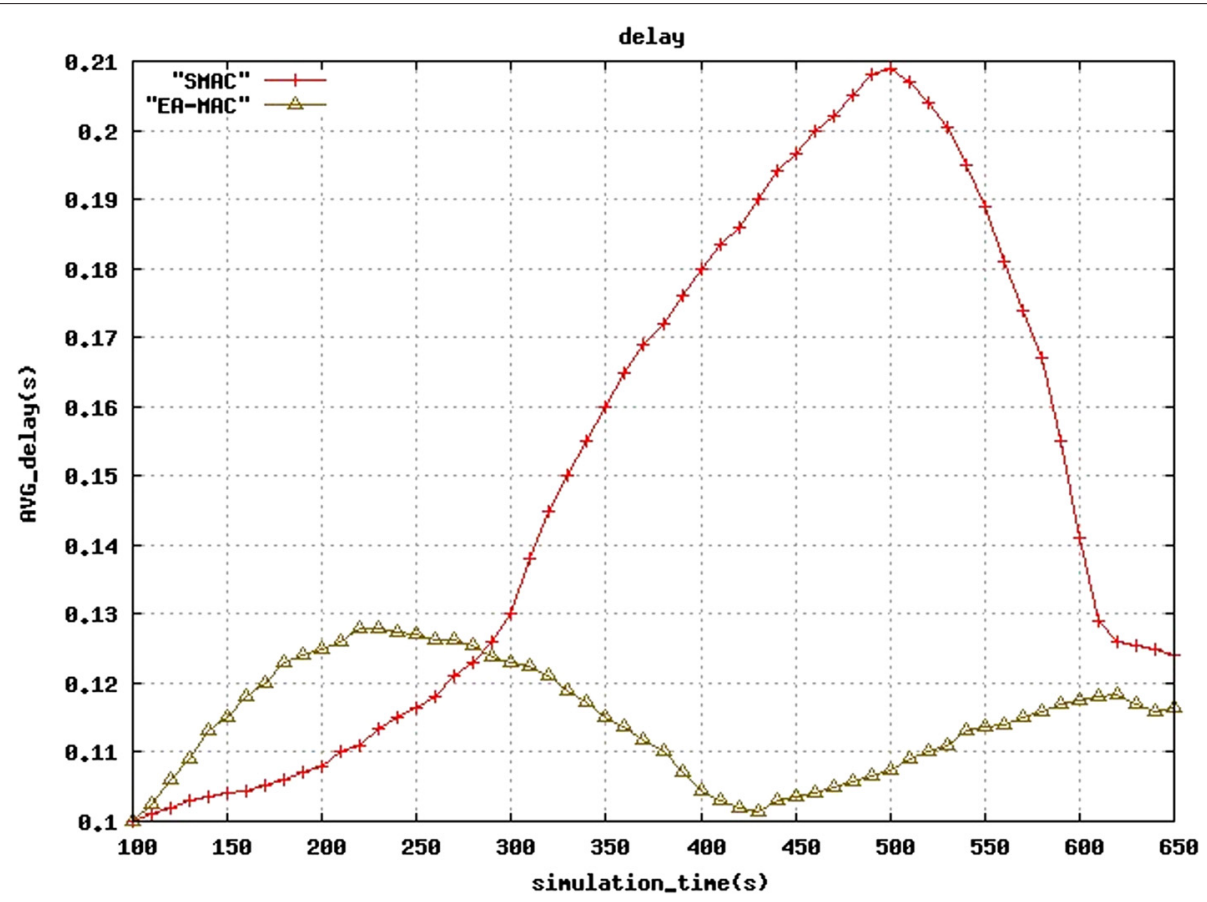

Fig. 8 Network delay

and adjusts the duty cycle. Before $450 \mathrm{~s}$, the packet loss rate of $S M A C$ increases continuously and reaches the climax in $450 \mathrm{~s}$. This is because $S M A C$ cannot timely process the previously lost data in $400 \mathrm{~s}$. So after $400 \mathrm{~s}$, even if networks restore the state of low traffic flow, packet loss rate cannot decrease timely.

\section{Conclusions}

In this paper, an improved algorithm of $E A-M A C$ protocol is given to address the deficiencies of the $S M A C$ protocol. Firstly, an algorithm for correlation computing between nodes is proposed which divides similar nodes to a certain area and chooses the representative node for

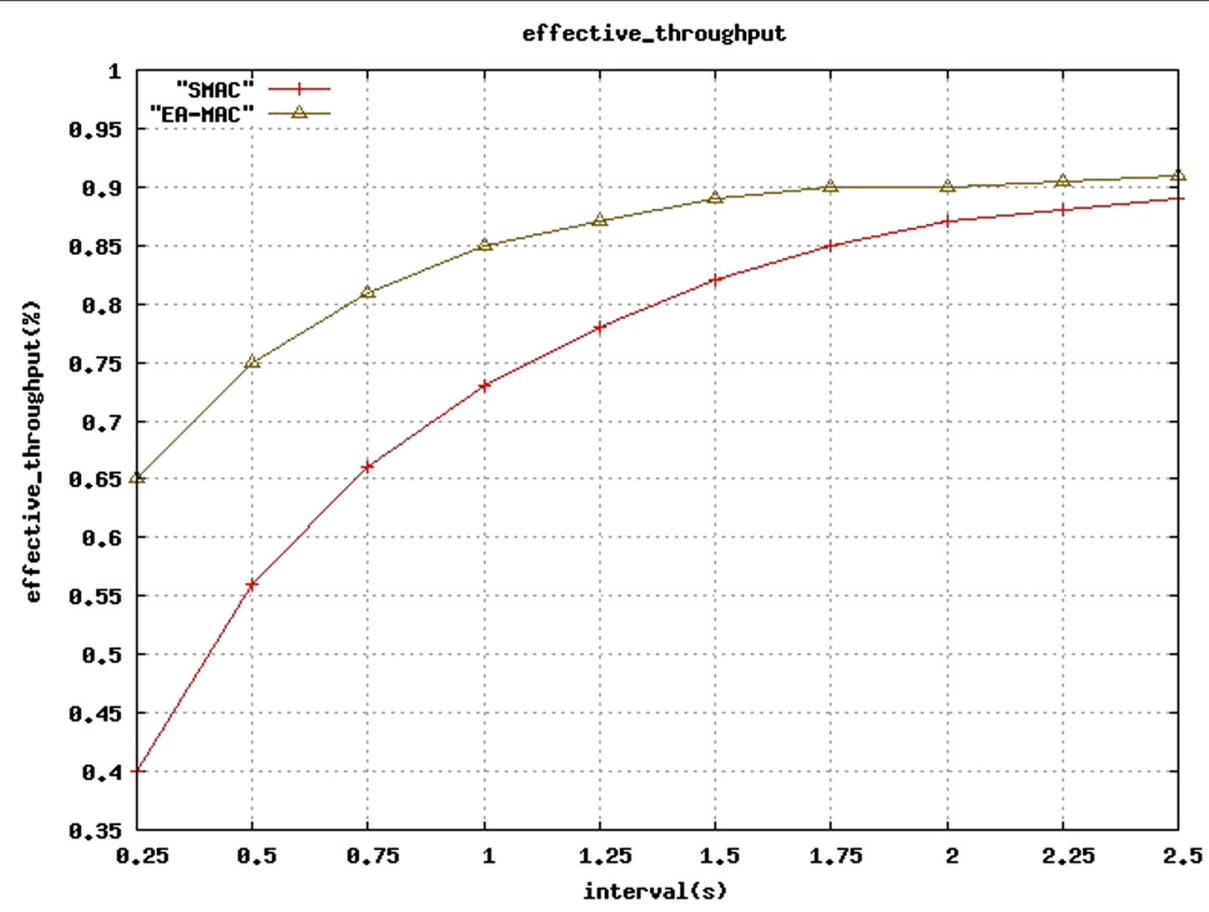

Fig. 9 Effective throughput 


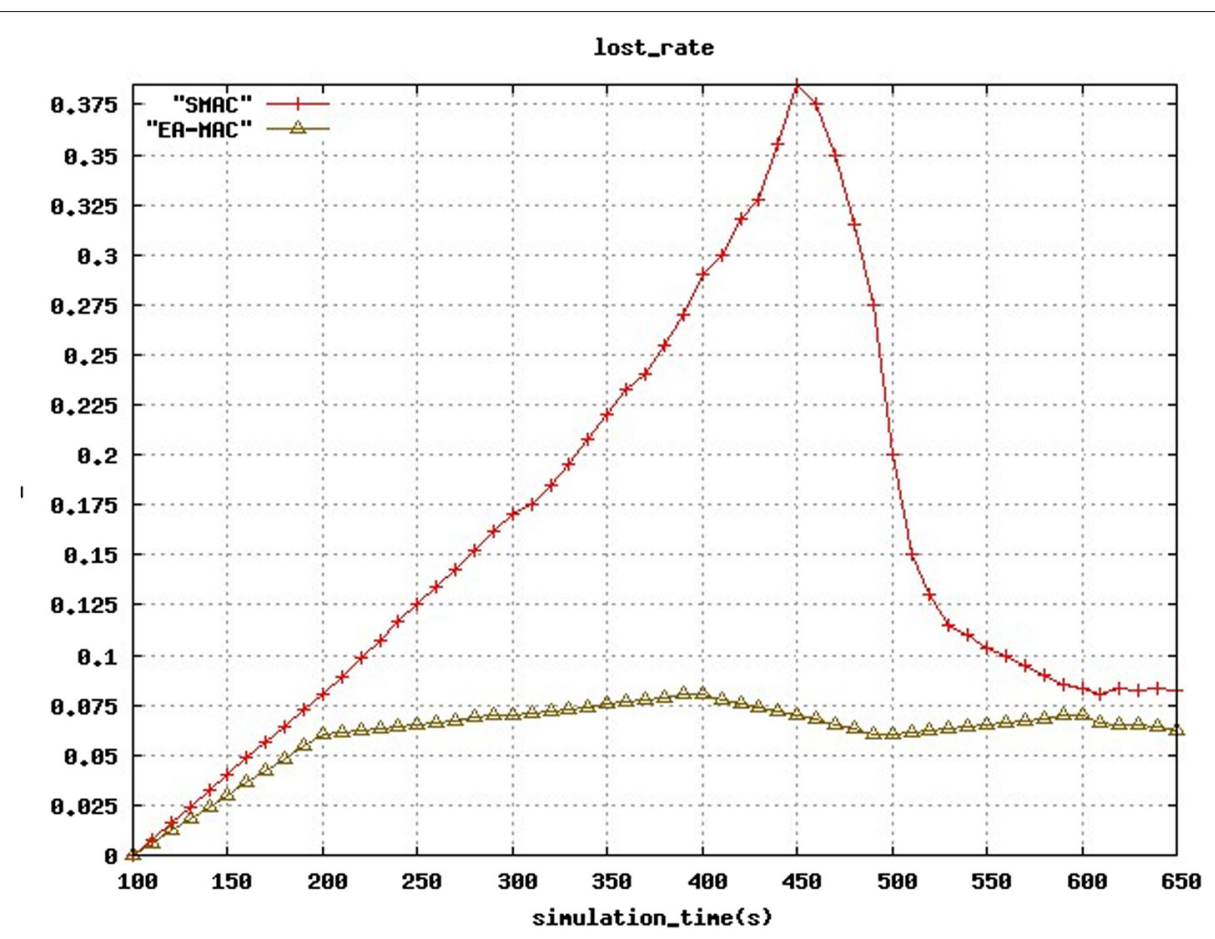

Fig. 10 Packet loss rate

data transmission in the considered region. Representative node is cyclically elected upon some parameters such as node residual energy and distance to sink node. In the transmission, network traffic prediction is conducted by $A R M A$ mode and the duty cycle is dynamically changed accordingly through comparing the predicted value with the pre-setting threshold value. Conflict probability is significantly controlled by altering the back-off range and constraining the back-off window size, effectively reducing energy consumption. Finally, NS2 is adopted to implement for simulations of $E A-M A C$ protocol and SMAC protocol. Simulation results including the network average energy consumption, latency and effective throughput, and packet loss rate are analyzed, showing that $E A-M A C$ protocol performs superior to that of $S M A C$ protocol.

\section{Competing interests}

The authors declare that they have no competing interests.

\section{Acknowledgements}

This work was supported by "the Fundamental Research Funds for the Central Universities" (2013QNB15) and the National Science Foundation of China under grant no. 61203304. Thanks for the contributors on this paper.

\section{Author details}

${ }^{1}$ School of Computer Science and Technology, China University of Mining and Technology, 221116 Xu Zhou, China. ${ }^{2}$ Visiting Research to the Department of Computing and Software, McMaster University, L8S 4K1 Hamilton, Canada.

Received: 21 September 2015 Accepted: 27 November 2015

Published online: 09 December 2015

\section{References}

1. H-c Lee, J-w Lee, J-h Hwang, H Yoe, in Future Generation Information Technology. A study on energy efficient MAC protocol of wireless sensor network for ubiquitous agriculture (Springer, Berlin Heidelberg, 2010), pp. 642-650

2. R Sokullu, E Demir, MAC layer protocols for linear wireless sensor networks: a survey. Commun. Special Issue on Ad-hoc Netw. 38(4), 393-422 (2014)

3. W-M Song, Y-M Liu, S-E Zhang, in Wireless Communications, Networking and Mobile Computing, 2008. WiCOM'08. 4th International Conference on. Research on SMAC protocol for WSN (IEEE, 2008), pp. 1-4

4. J Luo, L Jiang, C He, An analytical model for SMAC protocol in multi-hop wireless sensor networks. Sci. China Inf. Sci. 53(11), 2323-2331 (2010)

5. Q Wang, K Jaffrès-Runserb, J-L Scharbarg, C Frabou, Y Suna, J Lia, Z Lia, A thorough analysis of the performance of delay distribution models for IEEE 802.11 dcf. Ad Hoc Networks. 24, 21-33 (2014)

6. Y Yao, Q Cao, AV Vasilakos, in Mobile ad-hoc and sensor systems (MASS), 2013 IEEE 10th international conference on. EDAL: An energy-efficient, delay-aware, and lifetime-balancing data collection protocol for wireless sensor networks (IEEE, 2013), pp. 182-190

7. Y Yao, Q Cao, AV Vasilakos, EDAL: an energy-efficient, delay-aware, and lifetime-balancing data collection protocol for heterogeneous wireless sensor networks. IEEE/ACM Trans. Networking. 23, 810-823 (2015)

8. D Xu, K Wang, Stochastic modeling and analysis with energy optimization for wireless sensor networks. Int. J. Distrib. Sensor Netw. 2014, 1-5 (2014)

9. K Han, J Luo, Y Liu, AV Vasilakos, Algorithm design for data communications in duty-cycled wireless sensor networks: a survey. Commun. Mag. IEEE. 51(7), 107-113 (2013)

10. Y Xiao, M Peng, JH Gibson, GG Xie, DZ Du, AV Vasilakos, Tight performance bounds of multihop fair access for MAC protocols in wireless sensor networks and underwater sensor networks. Mob. Comput. IEEE Trans. 11(10), 1538-1554 (2012)

11. S Sengupta, S Das, M Nasir, AV Vasilakos, W Pedrycz, An evolutionary multiobjective sleep-scheduling scheme for differentiated coverage in wireless sensor networks. IEEE Trans. Syst. Man \& Cybern. Part C Appl \& Rev. 42(6), 1093-1102 (2012)

12. XY Liu, Y Zhu, L Kong, C Liu, Y Gu, AV Vasilakos, MY Wu, CDC : compressive data collection for wireless sensor networks. IEEE Trans. Parallel \& Distribut. Syst. 26, 2188-2197 (2015) 
13. $\mathrm{XXu}$, R Ansari, A Khokhar, AV Vasilakos, Hierarchical data aggregation using compressive sensing (HDACS) in WSNs. ACM Trans. Sensor Netw. (TOSN). 11(3), 45 (2015)

14. Y He, J Sun, X Ma, AV Vasilakos, R Yuan, W Gong, Semi-random backoff: towards resource reservation for channel access in wireless LANs. Netw. IEEE/ACM Trans. 21(1), 204-217 (2013)

15. Y Zeng, K Xiang, D Li, AV Vasilakos, Directional routing and scheduling for green vehicular delay tolerant networks. Wirel. netw. 19(2), 161-173 (2013)

16. S Coleri Ergen, C Fischione, C Borean, Method for setting the operation of a routing node of an asynchronous wireless communication network, network node and communication network implementing the method. (Google Patents, 2012). US Patent $8,243,620$

17. J Vrhovec, D Rudel, AM Lebar, in XII Mediterranean Conference on Medical and Biological Engineering and Computing 2010. The importance of uterine contractions extraction in evaluation of the progress of labour by calculating the values of sample entropy from uterine electromyogram (Springer, Berlin Heidelberg, 2010), pp. 140-143

18. K Mohammadi, HR Eslami, R Kahawita, Parameter estimation of an ARMA model for river flow forecasting using goal programming. j hydrol J. Hydrol. 331(1), 293-299 (2006)

19. C Dong, C Shao, SH Richards, LD Han, Flow rate and time mean speed predictions for the urban freeway network using state space models. Transp. Res. Part C Emerg. Technol. 43 part 1, 20-32 (2014)

20. $\mathrm{H}$ Gao, F Liu, in International Conference on Transportation Engineering 2009. A combined model for traffic flow prediction based on wavelet analysis (ASCE, 2009), pp. 2389-2394

21. Y Yang, Z Fu, TS Lee, MS Park, Tasl: A traffic-adapted sleep/listening MAC protocol for wireless sensor network. JPS. 2(1), 39-43 (2006)

22. M Moh, EJ Kim, TS Moh, Design and analysis of distributed power scheduling for data aggregation in wireless sensor networks. Int. J. Sensor Netw. 3-4, 143-15513 (2007)

23. J Lee, NT Dinh, G Hwang, JK Choi, C Han, Power-efficient load distribution for multihomed services with sleep mode over heterogeneous wireless access networks. IEEE Trans. Veh. Technol. 63(4), 1843-1854 (2014)

24. MJ Azhar, S Ko se, in Circuits and Systems (ISCAS), 2014 IEEE International Symposium On. An enhanced pulse width modulator with adaptive duty cycle and frequency control (IEEE, 2014), pp. 958-961

25. H Rasouli, YS Kavian, HF Rashvand, ADCA: adaptive duty cycle algorithm for energy efficient IEEE 802.15.4 beacon-enabled wireless sensor networks. Sensors J. IEEE. 14(11), 3893-3902 (2014)

26. P Shi, Y Wang, K Li, AT Chan, in Pervasive Computing and the Networked World. Cross-layer adaptive end-to-end delay control for asynchronous duty-cycle wireless sensor networks, (2014), pp. 520-531

27. KK Sharma, M Aggarwal, N Yadav, Squaring back off based media access control for vehicular ad-hoc networks, (2014), pp. 447-455

28. D Sharma, S Patsariya, A Mahajan, Effects of backoff factor on exponential backoff algorithm. MITA 2011, 326-329 (2011)

29. JL Huang, P Jin, LG Li, The Application of Binary Exponential Backoff Algorithm for WIA Wireless Network. Appl. Mech. Mater. 556, 4568-4572 (2014)

30. D Qiu, YX Yang, The Hidden Terminal Problem of 802.11 MAC and 802.15. 4 MAC Protocols Based on NS2 Platform. Advanced Mater. Res. 989 , 4078-4081 (2014)

31. KWu, H Dai, Z Yang, S Lu, D Chen, X Li, NDP2PSim: A NS2-based platform for peer-to-peer network simulations. Lect. Notes Comput. Sci. 3759 , 520-529 (2005)

32. N Zhao, X Li, M Yang, XT Huang, Vehicular ad-hoc network and routing design. Appl Mech \& Mater. 641-642, 829-832 (2014)

\section{Submit your manuscript to a SpringerOpen ${ }^{\circ}$ journal and benefit from:}

- Convenient online submission

- Rigorous peer review

- Immediate publication on acceptance

- Open access: articles freely available online

- High visibility within the field

- Retaining the copyright to your article

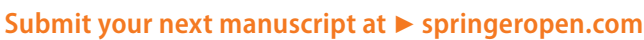

